\title{
Activación y determinación de parámetros cinéticos de la plasmina humana y Ovis aries
}

\section{Activation and comparative kinetics of Ovis aries plasmin with human plasmin}

\author{
Omaira Cañas B, ${ }^{1}$ BSc, Alfonso Quijano ${ }^{1}{ }^{1}$ Ph.D, Fernando Arbeláez R, ${ }^{1 *}$ Ph.D. \\ ${ }^{1}$ Universidad de Pamplona, Facultad de Ciencias Básicas, Grupo de Investigación en Química, Ciudadela \\ universitaria, Km 1, Vía Bucaramanga, Pamplona, Norte de Santander, Colombia. ${ }^{*}$ Correspondencia: \\ lui.ferar@hotmail.com
}

Recibido: Mayo de 2010; Aceptado: Diciembre de 2010.

\section{RESUMEN}

Objetivo. Comparar las cinéticas y activaciones, unificar las purificaciones y determinar las secuencias de los terminales- $\mathrm{N}$ de los plasminógenos Ovis aries y humano. Materiales y métodos. Los plasminógenos fueron purificados por el mismo método: cromatografías de afinidad e intercambio iónico, activados con urocinasa, la secuencia de los terminales- $\mathrm{N}$ se realizó por el método de Edman Resultados. La afinidad de la plasmina Ovis aries por el sustrato cromogénico fue de $0.45 \mathrm{mM}, 11.8$ veces mayor que la afinidad de la plasmina humana $(5.3 \mathrm{mM})$. Conclusiones. Se confirma y unifica el método de purificación de los plasminógenos del plasma, para todos los mamíferos. La alta afinidad de la plasmina Ovis aries confirma una mayor afinidad de las plasminas animales por el sustrato cromogénico, en comparación con la plasmina humana.

Palabras clave: Plasmina, Glu-Plasminógeno, fibrinólisis, urocinasa, cinética. (Fuente: MeSH)

\section{ABSTRACT}

Objective. Compare the kinetics and activations, unify the purifications methods and establish the sequence of the $\mathrm{N}$-terminals of the plasminogens human and Ovis aries. Materials and methods. The plasminogens were purified by the same methods: affinity and ion exchange chromatographies, furthermore both plasminogen were activated by human urokinase and the N-terminals sequence perform by the Edman's degradation's method. Results. The affinity of Ovis aries plasmin for the chromogenic substrate was determined in $0.45 \mathrm{mM}, 11.8$ times more affinity that the human plasmin $(5.3 \mathrm{mM})$. Conclusions. The method of the plasminogens purification and the unification was established for all mammals. The high affinity of the Ovis aries plasmin confirmed a mayor affinity of animal's plasmin to the chromogenic sustrate, compared with the human plasmin.

Key words: Plasmin, Glu-Plasminogen, fibrinolysis, urokinase, kinetics. (Source: MeSH) 


\section{INTRODUCCIÓN}

El sistema hemostático involucra el mantenimiento de la sangre en un estado fluido bajo condiciones fisiológicas y también reacciona rápidamente frente a la formación del coágulo de fibrina. La trombosis se considera la causa primaria de ataques al corazón y ocurre cuando los sistemas de coagulación y fibrinólisis pierden su equilibrio natural (1). La formación del coagulo puede ser iniciada por dos vías, la extrínseca y la intrínseca. La primera, ocurre cuando el factor VII (FVII), se une al receptor de membrana del factor tisular (FT) (2). Autoactivandose a FVII activado (FVIIa), a su vez el complejo formado FVIIa-FT, activa el factor X (FX), a FX activado ( $F X a)$. La vía intrínseca se inicia por el complejo kalicreina-factor XII, complejo que a su vez activa el factor XI (FXI), esta cascada conduce a la activación del FX, donde se encuentran ambas vías. Este último factor se convierte en la llave enzimática común de ambas vías, además en asociación con el factor $\mathrm{V}$ (FV), forman la protrombinasa, la cual convierte la protrombina en trombina, está última actúa sobre el fibrinógeno hidrolizando los fibrinopéptidos A y B, iniciando así la formación de la malla de fibrina (2).

En la vía intrínseca, el FXI es además activado directamente por la trombina formada, potenciando el sistema de coagulación, ya que este a su vez activa al factor IX (FIX) (3), lo cual finalmente forma la malla de fibrina.

El sistema fibrinólitico, es dependiente de la conversión del zimógeno plasminógeno (Plg), a la serino proteasa plasmina (Pli), por los activadores fisiológicos específicos, como el activador del Plg tipo urocinasa (u-PA), o el activador del Plg tipo tisular (t-PA) (4). El hígado es el tejido primario que sintetiza el Plg, sin embargo hay otras fuentes donde ha sido identificada esta proteína como las glándulas adrenales, riñones, entre otros órganos (5). La conversión del Plg a Pli, involucra el rompimiento de una sola secuencia (Arg561-Val562), resultando en la generación de la Pli, compuesta por dos cadenas, una de 561 aminoacidos (a.a.) pesada (A) y la otra liviana (B) de 230 a.a, unidas por un puente de disulfuro. La tríada catalítica está localizada en la cadena B, y consiste de His603, Asp646 y Ser741. En la cadena $A$, se encuentran los sitios de unión a la lisina (LBS) que van desde el a.a. (Glu1 Humano o Asp1 animales) hasta Arg561, las cuales están unidas por puentes de disulfuro y son los lugares por los cuales se fija la Pli a la fibrina específicamente para degradar el coagulo (6).

El complejo formado entre enzima-sustrato y la concepción del estudio cinético fue propuesto por Michaelis-Menten (7) y ha sido estudiada en otros Plgs por Cañas et al (8-9). Además se han realizado estudios sobre las características del Plg bufalino (10).

En este estudio se compara la activación y cinética de la Pli Ovis aries con la de la Pli humana, ampliando los conocimientos sobre el sistema fibrinolítico de estas especies mamíferas, contribuyendo a un mejor manejo de los problemas cardiovasculares, que cada día se constituyen en una patología que ocupa el primer lugar en el listado de causas de muerte en muchos países del mundo.

El objetivo de este trabajo fue comparar la cinética y activación de los Plgs Ovis aries y humano, relacionando estos parámetros con el funcionamiento del sistema fibrinólitico de ambas especies.

\section{MATERIALES Y MÉTODOS}

Toma y procesamiento de las muestras de plasma. Este estudio se hizo bajo las recomendaciones establecidas en la normatividad sobre investigación con seres humanos (Declaración Universal de los derechos Humanos, Helsinki-Tokio y Nuremberg) con el aval del comité de Bioética de la Universidad de Pamplona. Se tuvo en cuenta el criterio de la utilización del consentimiento informado a la hora de la extracción del plasma, establecido en el Art, 30, 08430. 
El plasma Humano fue suministrado por el Hospital Erasmo Meoz de la ciudad de Cúcuta-Colombia, analizado y certificado libre de enfermedades infecciosas.

La muestra del plasma Ovis aries, fue tomada con la autorización del comité de Bioética de la Universidad de Pamplona en la Granja Experimental Villa Marina, también propiedad de dicha institución, por un médico veterinario, siguiendo las recomendaciones contempladas en él título $\mathrm{V}$ del decreto 08430. Se utilizaron bolsas para extracción sanguínea con solución anticoagulante de citrato, fosfato, dextrosa y adenina (CPDA-1). A la sangre obtenida de esta manera, se le añadió fenilmetilsulfonilfluoruro (PMSF) disuelto en dimetil-sulfoxido (DMSO), a una concentración final de $1 \mathrm{mM}$, como inhibidor de la actividad de las serino proteasas. Las muestras se mantuvieron refrigeradas y el plasma separado por centrifugación a 7.000 r.p.m. durante $15 \mathrm{~min}$. a $4^{\circ} \mathrm{C}$.

Reactivos. PMSF, $\varepsilon$-amino ácido caprioico ( $\varepsilon-A C A)$ y ácido clorhídrico de (Fabroquim), cloruro de sodio $(\mathrm{NaCl})$, DMSO y acetato de sodio anhidro de (Riedel-de-Haën), $\mathrm{N}, \mathrm{N}^{\prime}$-Metilen-bisacrilamida, persulfato de amonio, $\beta$-mercaptoetanol, sodio dodecyl sulfato (SDS), di-hidrógeno fosfato de sodio dihidratado de (Merck), lisinasefarosa-4 $\beta$ de (Amersham Biosciences), ácido acético concentrado de (Baker), sustrato cromogénico para Pli Spectrozyme y urocinasa (u-PA) $(110.000 \mathrm{UI} / \mathrm{ml})$ de (Amdiag). El sustrato cromogénico S-2251, se disolvió a una concentración de $5 \mathrm{mM}$, concentraciones crecientes del mismo, el estándar de peso molecular usado fue: $180 \mathrm{kDa}$ (a2-macroglobulina), 92KDa (Glu-Plg), 66 KDa (cadena-a, fibrinógeno humano), $52 \mathrm{KDa}$ (cadena- $\beta$, fibrinógeno humano), $46 \mathrm{KDa}$ (cadena- $y$, fibrinógeno humano), $23.8 \mathrm{kDa}$ (Tripsina), y agua tipo I y III, de los (Laboratorios de Investigación en Química-Universidad de Pamplona).

Purificación de los plasminógenos. LoS Plgs de las dos especies fueron purificados por cromatografía de afinidad y exclusión según el método de Cañas et al (8). En una columna de $8 \times 2.5 \mathrm{~cm}$ (Biorad), equilibrada con tres volúmenes de solución tampón de $0.1 \mathrm{M}$ fosfato $0.15 \mathrm{M}$ de $\mathrm{NaCl} \mathrm{pH} 7.3$ (PBS), a una velocidad de flujo de $2 \mathrm{ml} /$ min. Se aplicaron $200 \mathrm{ml}$ de plasma para cada especie, posteriormente la columna se lavó con PBS, hasta que la absorbancia A280 fue $\leq 0.01$, se eluyó con $100 \mathrm{ml}$ de PBS que contenían 0.05M $\varepsilon$-ACA y colectado en fracciones de $3 \mathrm{ml}$ por tubo, la concentración fue determinada utilizando el coeficiente absorción de $(\varepsilon 1 \%) 1 \mathrm{~cm}=$ 1.6 (mol-1 cm-1) (11).

Los Plgs obtenidos, fueron separados por cromatografía de intercambio iónico según el método de Cañas et al (8). En una columna de $5 \times 2.5 \mathrm{~cm}$ (Biorad), empacada con $4 \mathrm{ml}$ de DEAE sefarosa y equilibrada con la solución tampón 0.06 $M$ Tris, $0.06 \mathrm{M} \mathrm{NaCl}$. $0.02 \mathrm{M} \mathrm{HCl} \mathrm{pH} 8.5$ (A). Sé eluyó con un gradiente lineal usando la solución tampón $\mathrm{A}$ y $0.07 \mathrm{M}$ Tris, $0.22 \mathrm{M} \mathrm{NaCl}, 0.06 \mathrm{M} \mathrm{HCl} \mathrm{pH}$ 7.5.(B) Fue colectada en fracciones de $3 \mathrm{ml}$ a una velocidad de flujo de $1.5 \mathrm{ml} / \mathrm{min}$.

Análisis electroforético. Las electroforesis SDS-PAGE se realizaron de acuerdo al método de Laemmli (12). Las muestras de Plgs de $5 \mu \mathrm{g}$ fueron mezcladas con el buffer de muestra en un volumen $1: 1$ (vol/vol), fueron tratadas térmicamente por 5 minutos a $100^{\circ} \mathrm{C}$ y visualizadas por tinción de azul brillante de Coomassie.

Transferencia directa de los PIgs humano y ovis aries a una membrana de (PVDF). Se tomaron $2 \times 2 \mu \mathrm{l}$ de las dos proteínas y se diluyeron en $2 \times 500 \mu \mathrm{l}$ de ácido acético al $0.1 \%$, en agua destilada tipo I en dos tubos para microcentrifuga. Se tomaron trozos de membrana PVDF de un tamaño no mayor a $3 \times 3 \mathrm{~mm}$, y se humedecieron con metanol al $99 \%$ durante 15 segundos. Se añadió un trozo de membrana PVDF totalmente húmeda con metanol, se agitó suavemente durante 3 minutos y se mantuvo a una temperatura de $5-8^{\circ} \mathrm{C}$ agitando de igual manera cada 8-12 horas, luego de 2 días las membranas se secaron y se lavaron 2 veces con metanol en agua destilada al 20\%. A las proteínas se les determinó la secuencia del terminal-N según el método de Edman 
(13), en la universidad de Umeå Suecia.

Activación de los plasminógenos. Se utilizó $1 \mathrm{mg}$ para cada uno de los Plgs a una concentración de $1 \mathrm{mg} / \mathrm{ml}$ y a una temperatura constante de $37^{\circ} \mathrm{C}$, a los cuáles se les adicionó $6.72 \mu$ le u-PA para una concentración final de 739.2 $\mathrm{UI} / \mathrm{ml}$ "Solución activada". Sé monitoreó espectrofotométricamente (A405) así: Se tomaron 8 tubos de sustrato $(0.3 \mathrm{mM})$ equivalentes a los diferentes tiempos de incubación, $(0,1,3,6,9,15,25$ y 35 minutos), y se les adicionaron $3 \mu$ de la "Solución activada", se dejó reaccionar por 12 segundos, la reacción se detuvo con la adición de $10 \mu \mathrm{l}$ de la solución tampón acetato (BA) $4 \mathrm{M} \mathrm{pH} 3.8$.

Al minuto 35, la activación de los Plgs se detuvo adicionando a las soluciones del "Plg activado", $323 \mu$ lde glicerol al 100\%, para una concentración de glicerol final de $25 \%$. La solución activada, se homogenizó, fraccionó y almacenó a $-80^{\circ} \mathrm{C}$. Se determinó la $A 405$ de las 8 incubaciones, y sé gráfico la A405 vs tiempo de incubación.

Determinación de la concentración de plasmina. De acuerdo al proveedor del sustrato cromogénico, 10 miliabsorbancias $\left(\mathrm{mA}_{405}\right)$ a $37^{\circ} \mathrm{C}$ corresponde a $1 \mathrm{nM}$ de Pli (14).

A los tubos con sustrato $0.3 \mathrm{mM}$, se les agregó $3 \mu \mathrm{l}$ de la solución final activada y se incubaron por 0, 30 y 60 segundos respectivamente, la reacción se detuvo adicionando $10 \mu \mathrm{l}$ de BA, la $A_{405}$ se determinó para cada tubo y se determinaron las $\mathrm{mA}_{405} / \mathrm{min}$.

Cinética de la plasmina. Se estudia la cinética de las dos especies de Plis partiendo de una concentración común $(2.7 \mu \mathrm{M})$, a una temperatura de $37^{\circ} \mathrm{C}$ y un $\mathrm{pH} 7.3$, su actividad se determinó por la velocidad de transformación del sustrato a producto, a $\left(\mathrm{A}_{405}\right)$, utilizando las siguientes concentraciones de sustrato: $0.05,0.08$, $0.10,0.13,0.18,0.25,0.30 \mathrm{mM}$, y adicionándoles $3 \mu$ l de cada $\mathrm{Pli}$, la reacción se detuvo con $10 \mu$ de BA, la A405 se determinó y se gráfico según la ecuación de Lineweaver-Burk (15).

\section{RESULTADOS}

Análisis electroforético. La pureza del Plg Ovis aries se determinó mayor al $95 \%$ (Figura 1, carril 2) esta banda migra aproximadamente a la misma altura que el Plg humano utilizado en el marcador de peso molecular, carril 1 banda 2 de $92 \mathrm{kDa}$.

Activación de los plasminógenos. En la figura 2, carril 2, se visualiza la clásica forma de activación del Plg humano a Pli, formando las típicas bandas, A y B a los 35 minutos de incubación. Se observa en el mismo carril que al minuto 35, la conversión del zimógeno a cadena liviana y pesada es del $100 \%$ en el Plg humano. Se ve una acción autodegradativa del Plg Ovis aries carril 3, al comparar con el carril 2 del humano, el Ovis aries al minuto 35, todavía posee moléculas de Plg por activar (92 kDa), también se observa en la banda B y A del plg humano, cierta degradación por la misma acción de la $\mathrm{Pli}$, formando otras bandas diferentes a la A y B (Figura 2 carril 2). La conversión de los Plgs a Pli,

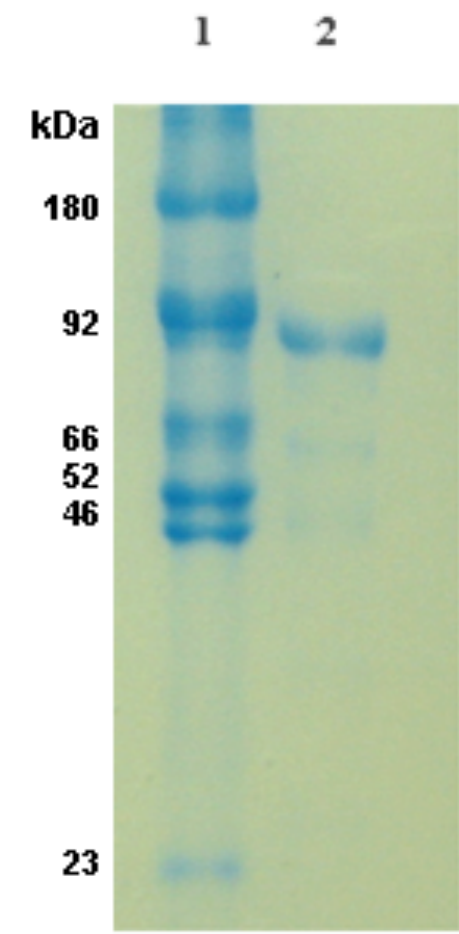

Figura 1. Electroforesis SDS-PAGE al 10\%, de la purificación del plg Ovis aries. Carril 1 marcador de peso molecular (ver materiales y métodos) carril 2 Plg Ovis aries. 


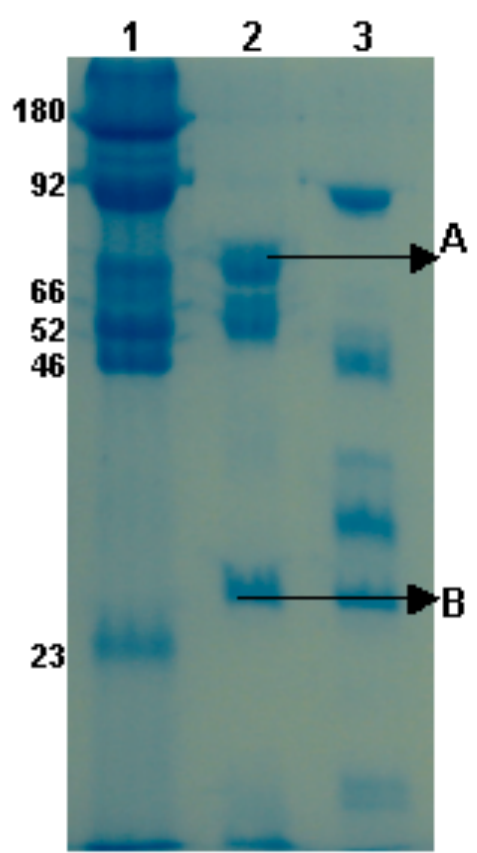

Figura 2. Electroforesis SDS-PAGE, 10\%, activación de los Plgs humano y Ovis aries a Pli. Carril 1, marcador de peso molecular, carriles 2 y 3, Plis humano y Ovis aries, respectivamente al minuto 35. Se indica la migración de la cadena pesada (A), y liviana (B), para las dos Pli.

por activación con u-PA fue monitoreado de acuerdo a la cantidad de sustrato hidrolizado y detectado a $A_{405 \mathrm{~nm}}$ (Figura 3 ).

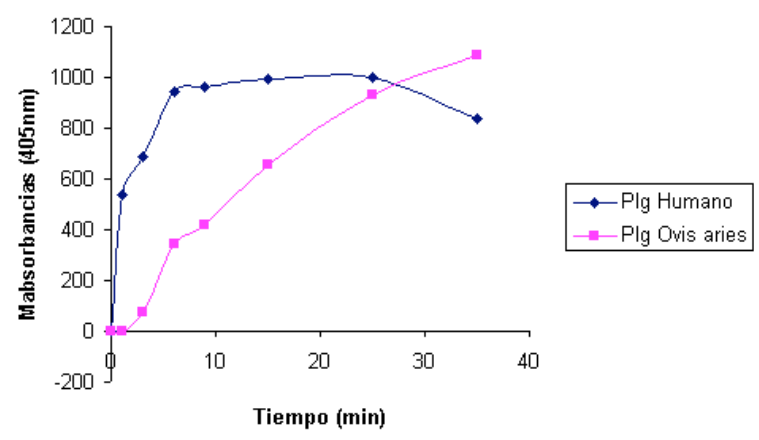

Figura 3. Monitoreo espectrofotométrico A405 de la activación de los Plgs Ovis aries y humano a Pli por u-PA. A405 vs tiempo de incubación.

Cinética de la plasmina. Los parámetros cinéticos de las dos Plis fueron determinados en la figura 4 ( $A$ y $B$ ), en las coordenadas de Lineweaver-Burk, donde se observa el comportamiento de las Plis. Los resultados
A. Cinética de la Plasmina Humana

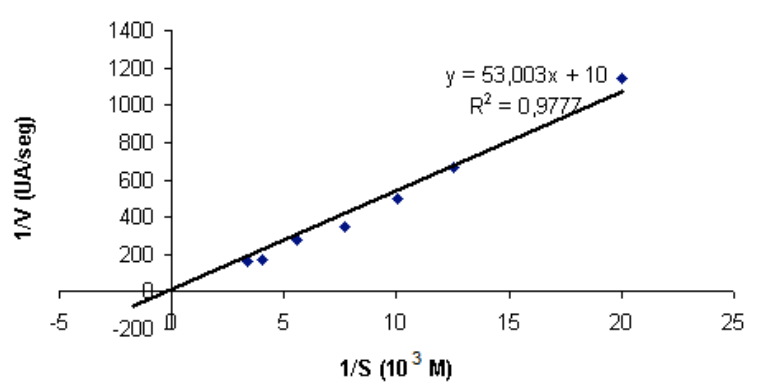

B. Cinética de la Plasmina Ovis aries

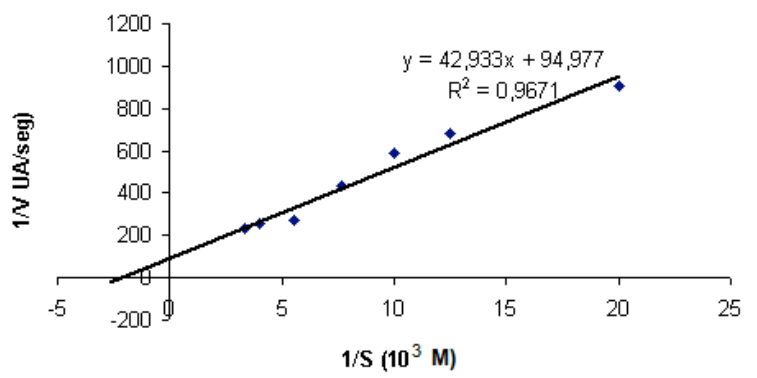

Figura 4. Dependencia de la velocidad de transformación del sustrato a producto por las Plis humana y Ovis aries en las coordenadas de la ecuación de Lineweaver-Burk.

de los parámetros de la cinética de la Pli Ovis aries se resumen (Tabla 1 , columna 3), comparandose con los parámetros de la Pli humana (Tabla 1, columna 2). Los parámetros de importancia cinética de las dos especies de Plis se resumen y comparan en la tabla 2.

Tabla 1. Velocidades (V) de las Plis humana y Ovis aries en función de concentraciones ascendentes de sustrato.

\begin{tabular}{ccc}
\hline \multicolumn{3}{c}{ 1/N UA*/seg. } \\
$1 /[\mathrm{S}] 10^{3} \mathrm{M}$ & Pli Humana & Pli Ovis aries \\
\hline 20 & 1144 & 907 \\
12.5 & 668 & 682 \\
10 & 496 & 587 \\
7.7 & 346 & 436 \\
5.5 & 277 & 270 \\
4 & 274 & 259 \\
3.33 & 160 & 232 \\
\hline
\end{tabular}

* Unidades arbitrarias/segundos. 
Tabla 2. Parámetros cineticos determinados según la ecuación de LineweaverBurk para las dos especies de Plis.

\begin{tabular}{cccc}
\hline \multicolumn{1}{c}{ Pli } & $\begin{array}{c}\text { Vmáx } \\
\text { UA/seg. }\end{array}$ & $\mathrm{KM} \mathrm{mM}$ & $\begin{array}{c}\mathrm{K} \text { cat UA seg- } \\
1 / \mu \mathrm{M}\end{array}$ \\
\hline Humana & 0.1 & 5.3 & $3.7 \times 10-2$ \\
Ovis aries & 0.010 & 0.45 & $3.70 \times 10-3$ \\
\hline
\end{tabular}

Secuenciamiento del terminal-N de los PIgs humano y Ovis aries. La secuencia del terminal-N de ambas especies de Plgs se aprecia en la tabla 3, las diferencias entre las dos especies se muestran en negrilla.

Tabla 3. Determinaciones de las secuencias de los Terminales-N de los Plg Humano y Ovis aries. Las diferencias en a.a. se indican en negrilla.

\begin{tabular}{cc}
\hline Plg & Secuencia Terminal-N \\
\hline Glu-Plg(Humano) & EPLDDY \\
D-Plg (Ovis aries) & DPPDDY \\
\hline
\end{tabular}

\section{DISCUSIÓN}

La purificación del Plg de muchos mamíferos se ha realizado por el mismo método $(8,9)$. El aislamiento del $\mathrm{Plg}$ de Ovis aries del plasma de este, demuestra definitivamente que el Plg de los mamíferos y quizás de cualquier especie se pueda purificar por el método reformado de Cañas et al (8) unificando así la purificación de esta molécula, la alta pureza de la purificación fue confirmada por las secuencias de los Plgs, ya que solo se encontró una sola secuencia en cada preparación, se debe tener en cuenta que el método utilizado para la determinación del terminal- $\mathrm{N}$, detecta cantidades tan bajas como 1 nanogramo de proteína. Estas secuencias se diferenciaron entre las dos especies en solo dos a.a., resultados que están en concordancia con otros estudios realizados (8-10). Los $\mathrm{Plg}$ de las dos especies en estudio, fueron activados, demostrándose que el plg del humano se activa totalmente formando las típicas bandas pesada y liviana, mientras que el Plg del Ovis aries, mostró una resistencia a la activación total, aunque las cadenas pesada y liviana se degradan más rápidamente, que la humana.

La afinidad de estas dos Plis, por el sustrato, también sigue los parámetros, determinados y comparados entre diferentes mamíferos irracionales y el humano (8-10), siendo la afinidad de las Plis de los animales la más alta por el sustrato cromogénico, hecho este que facilitará el estudio de los sistemas de coagulación y fibrinólisis entre los mamíferos y otras especies.

En este estudio se confirma una vez mas que esta tendencia se mantiene al ser determinada la afinidad para el Ovis aries en $0.45 \mathrm{mM}$. Las diferentes afinidades de los mamíferos podría interpretarse como diferentes capacidades de degradar el trombo y la formación o acumulación de fibrina en la circulación de estos mamíferos a su vez, diferentes capacidades de deshacerse de los trombos formados, siendo el humano de acuerdo con estos resultados, el más expuesto a sufrir problemas cardiovasculares.

La realidad mundial ha demostrado que la primera causa de muerte en humanos, son los problemas cardiovasculares (16). Puede que el ser humano este sometido a un sin número de factores extras que conllevan a problemas cardiovasculares como; estrés, alimentación, genética, tabaquismo, entre otros. El estudio multifactorial de las causas de los problemas cardiovasculares del ser humano y su comparación con los mamíferos más cercanos al ser racional no será fácil, pero en ese estudio y otros se deberá tener en cuenta el hecho de que la molécula del $\mathrm{Plg} / \mathrm{Pli}$ tiene mayor afinidad hacia el trombo en los animales que en el humano.

Finalmente se debe profundizar más en el estudio de esta molécula y la utilización del Plg/Pli animales en el ámbito clínico y terapéutico. Esto cobra importancia ya que uno de los pasos tempranos de la formación del trombo, es la hidrolización de los fibrinopéptidos, $A \alpha$ y $B \beta$ (17), del fibrinógeno. Estos monómeros de fibrina 
aún solubles a bajas concentraciones plasmáticas, podrían ser detectadas por la alta afinidad hacia ellos de los Plg/Plis de los animales, previniendo o detectando tempranamente la formación o probabilidad de la formación del trombo.

En conclusión, se confirma y unifica el método de purificación de los plasminógenos del plasma, para todos los mamíferos. La alta afinidad de la plasmina Ovis aries confirma una mayor afinidad de las plasminas animales por el sustrato cromogénico, en comparación con la plasmina humana.

\section{Agradecimientos}

A los doctores Torgny Stigbrand y Per Ingvar Olhsson de la universidad de Umeå en Suecia por el soporte económico y la determinación de las secuencias. Al Hospital Universitario Erasmo Meoz de Cúcuta, Colombia, por la donación del plasma humano.

\section{REFERENCIAS}

1. Kleinschnitz $C$, Stoll $M$, Bendszus $\mathrm{K}$, Schuh $\mathrm{H}$, Pauer $\mathrm{P}$, Burfeind $\mathrm{C}$ et al. Targeting coagulation factor XII provides protection from pathological thrombosis in cerebral ischemia without interfering with hemostasis. J Exp Med 2006; 203:513-518.

2. Mackman N. Role of tissue factor in hemostasis thrombosis, and vascular development. Arterioscler Thromb Vasc Biol 2004; 24:1015-1022.

3. Whelihan MF, Orfeo T, Gissel MT, Mann KG. Coagulation procofactor activation by factor XIa. J Thromb Haemost 2010; 7:1532-1539.

4. Kolodziejczyk J, Wachowicz B. Dysfunction of fibrinolysis as a risk factor of thrombosis. Pol Merkur Lekarski 2009; 160:341-5.

5. Zhang L, Seiffert D, Fowler BJ, Jenkins GR, Thinnes TC, Loskutoff DJ, Parmer RJ, Miles LA. Plasminogen has a broad extrahepatic distributions. Thromb Haemost 2002; 87:493-501.

6. Kouemo S, McMillan E, Doctor V. Mechanism of the synergistic effect between oversulfated chondroitin-6sulfate and lysine or 6-aminohexanoic acid in enhancing the in-vitro activation of glutamic plasminogen by tissue plasminogen activator or urokinase. Blood Coagul Fibrinolysis 2010; 21:425-430.
7. Walter NG. Michaelis-Menten is dead, long live Michaelis-Menten!. Nat chem Biol 2006; 2:66-67.

8. Cañas O, Quijano A, Arbeláez LF. Activación y cinética comparativa de dos especies de Plasminógenos: Humano y Bovino. Bistua 2006; 4:3-12.

9. Cañas O, Quijano A, Arbeláez LF. Cinética comparativa de la Plasmina canina con la humana, bovina y equina. Bistua 2007; 5:17-24.

10. Cañas O, Quijano A, Arbeláez LF. Activación y Cinética comparativa de la Plasmina Bufalina con la humana. Rev Col Cien Pec 2010; 23:47-54.

11. Longstaff C, Whitton CM. Aproposed reference method for plasminogen activators that enables calculation of enzyme activities in SI units. J Thromb Haemost 2004; 2:1416-1421.

12. Chakavarti B, Chakavarti D. Electrophoretic separation of proteins. J vis Exp 2008; 16:3791-758.

13. Oe $T$, Maekawa $M$, Satoh $R$, Lee $\mathrm{SH}$, Goto T. Combining [13C6]phenylisothiocyanate and the Edman degradation reaction: a possible breakthrough for absolute quantitative proteomics together with protein identification. Rapid commun mass spectrom 2010; 2:173-9. 
14. Christensen B, Schack L, Kläning E, Sørensen ES. Osteopontin is cleaved at multiple sites close to its integrinbinding motifs in milk and is a novel substrate for plasmin and cathepsin D. J Biol Chem 2010; 285:7929-37.

15. Weinberg $M L$, Felicori LF, Bello CA, Magalhaes HP, Almeida AP, Magalhaes A, Sanchez EF. Biochemical properties of a bushmaster snake venom serine proteinase (LV-Ka) and its kinin releasing activity evaluated in rat mesenteric arterial rings. J Pharmacol Sci $2004 ; 96: 333-42$
16. Daviglus ML, Lloyd-jones DM, Pirzada A. Preventing cardiovascular disease in the 21st century: therapeutic and preventive implications of current evidence. Am J Cardiovasc Drugs 2006; 6:87-101.

17. Weisel JW. Fibrinogen and fibrin. Adv Protein Chem 2005; 70:247-99. 